\title{
Optimization over the Efficient Set: Four Special Cases
}

\author{
H. P. BENSON ${ }^{1}$ AND S. SAYMN ${ }^{2}$
}

\begin{abstract}
Recently, researchers and practitioners have been increasingly interested in the problem (P) of maximizing a linear function over the efficient set of a multiple objective linear program. Problem (P) is generally a difficult global optimization problem which requires numerically intensive procedures for its solution. In this paper, simple linear programming procedures are described for detecting and solving four special cases of problem (P). When solving instances of problem (P), these procedures can be used as screening devices to detect and solve these four special cases.
\end{abstract}

Key Words. Multiple criteria decision making, efficient set, global optimization, linear programming.

\section{Introduction}

The multiple objective linear programming problem involves the simultaneous maximization of $p \geqq 2$ noncomparable linear criterion functions over a nonempty polyhedron. The concept of an efficient solution has played a useful role in the study of this problem. Many of the approaches for analyzing multiple objective linear programs involve generating the efficient set or subsets of the efficient set. Included among these approaches, for instance, are the vector maximization approach, interactive approaches, and several others [see, for instance, Evans (Ref. 1), Goicoechea, Hansen, and Duckstein (Ref. 2), Rosenthal (Ref. 3), Sawaragi, Nakayama, and Tanino (Ref. 4), Stadler (Ref. 5), Steuer (Ref. 6), Yu (Refs. 7-8), Zeleny (Ref. 9) and references therein].

Recently, researchers and practitioners have been increasingly interested in the problem (P) of optimizing a linear function over the efficient set of a multiple objective linear program. This problem arose in response to some of the difficulties in using multiple objective linear programming as

\footnotetext{
${ }^{1}$ Professor, College of Business Administration, University of Florida, Gainesville, Florida. ${ }^{2}$ Graduate Assistant, College of Business Administration, University of Florida, Gainesville, Florida. Presently, Assistant Professor, School of Business Administration, Bilkent University, Ankara, Turkey.
} 
a decision aid. For instance, certain real-world problems can be more appropriately represented and more easily analyzed as optimizations over the efficient set than as multiple objective linear programs (Refs. 10-12). In addition, a special case of optimization over the efficient set, the minimization of a criterion function of a multiple objective linear program over the efficient set of the program, has several important uses in improving multiple objective linear programming procedures. It aids, for instance, in setting goals, in ranking objective functions, and in using interactive algorithms more effectively (Refs. 13-16).

Mathematically, the problem of optimizing over the efficient set can be classified as a global optimization problem, since the feasible region of this problem is, in general, a nonconvex set. Global optimization problems possess local optima, frequently large in number, which need not be globally optimal. Therefore, algorithms for globally solving such problems, when available, are generally quite numerically intensive (Refs. 17-18).

Some algorithms and algorithmic ideas for optimizing over the efficient set have been proposed. They invariably rely upon difficult search procedures or repeated solutions of global optimization subproblems. Philip (Ref. 12) and Bolintineanu (Ref. 19) have described procedures using local search and cutting planes which could potentially solve the problem. Benson (Refs. 20-21) has proposed two implementable relaxation algorithms for the problem. Due to the difficulty of the problem, some heuristic methods for approximating an optimal solution have also been proposed (Refs. 13, 22). None of these algorithms or heuristics attempts to detect special cases of the problem prior to the use of the main procedure.

In this paper, simple linear programming procedures are described for detecting and solving four special cases of problem (P). These special-case procedures require very little computational effort in comparison to that required by algorithms for the general problem $(\mathrm{P})$. Therefore, when solving problem $(\mathrm{P})$, we recommend that they be used as screening devices to detect and solve the four special cases.

In Section 2, some definitions and preliminary ideas are presented, including descriptions of the four special cases of optimization over the efficient set of concern in this paper. Section 3 gives some theoretical results. These results serve as a basis for some of the detection and solution procedures for the four special cases. In Section 4 the detection and solution procedures are described, and some conclusions are given in the last section.

\section{Definitions and Preliminaries}

Let $X \subseteq R^{n}$ be a nonempty, compact polyhedron in $R^{n}$. Assume that $p \geqq 2$ and that $c_{i}^{T} \in R^{n}, i=1,2, \ldots, p$, are row vectors. Let $C$ denote the 
$p \times n$ matrix whose $i$ th row equals $c_{i}^{T}, i+1,2, \ldots, p$. Then the multiple objective linear programming (MOLP) problem may be written as

(MOLP) $V \max C x, \quad$ s.t. $x \in X$.

The concept of an efficient solution has played a prominent role in analyzing problem (MOLP), where an efficient solution is defined as follows.

Definition 2.1. A point $x^{\circ}$ is an efficient solution of problem (MOLP) when $x^{\circ} \in X$ and, if $C x \geqq C x^{\circ}$ for some $x \in X$, then $C x=C x^{\circ}$.

An efficient solution is also called a nondominated or Pareto-optimal solution. Let $X_{E}$ denote the set of all efficient solutions of problem (MOLP).

The central problem of interest in this paper is the problem of optimizing a linear function over the efficient set $X_{E}$ of problem (MOLP). This problem, which we shall denote problem $(\mathrm{P})$, is given by

(P) $\max \langle d, x\rangle, \quad$ s.t. $x \in X_{E}$,

where $d \in R^{n}$ is nonzero. Let $v$ denote the optimal value of problem (P).

Let

$$
Y=\{C x \mid x \in X\} .
$$

Then, from Rockafellar (Ref. 23), $Y$ is a nonempty, compact polyhedron. To help analyze problem (P), we will be interested in the related multiple objective linear program (MOLPY) given by

(MOLPY) Vmax $I_{p} y, \quad$ s.t. $y \in Y$,

where $I_{p}$ denotes the $p \times p$ identity matrix. Let $Y_{E}$ denote the set of efficient solutions for this problem.

The concept of the ideal solution for problem (MOLPY) has proven useful in analyzing both problems (MOLP) and (MOLPY), where the ideal solution is defined as follows.

Definition 2.2. A point $y^{I} \in R^{p}$ is called the ideal solution for problem (MOLPY) when, for each $i=1,2, \ldots, p$,

$$
y_{i}^{\prime}=\max y_{i}, \quad \text { s.t. } y \in Y .
$$

Notice that the ideal solution $y^{I}$ for problem (MOLPY) need not belong to $Y$. However, when $y^{I} \in Y$, then $Y_{E}=\left\{y^{n}\right\}$. Thus, it is clear that the case of $y^{I}$ belonging to $Y$ is a special case of problem (MOLPY). 
Another special case for problem (MOLPY) is given in the following definition.

Definition 2.3. Problem (MOLPY) is said to be completely efficient when $Y=Y_{E}$.

Similarly, when $X=X_{E}$, problem (MOLP) is called completely efficient. Notice that problem (MOLPY) is completely efficient if and only if problem (MOLP) is completely efficient.

To help analyze problem (P), we will also be interested in the linear programming relaxation of problem $(\mathrm{P})$. This problem, which we will denote problem $\left(L P_{U B}\right)$, is given by

$\left(\mathrm{LP}_{\mathrm{UB}}\right) \max \langle d, x\rangle, \quad$ s.t. $x \in X$.

Let $U B$ denote the optimal objective function value of problem ( $\left.L P_{U B}\right)$. Then $U B$ is a finite number and gives an upper bound on the optimal objective function value $v$ of problem (P).

Let $X_{\mathrm{ex}}$ and $Y_{\mathrm{ex}}$ denote the sets of extreme points of the polyhedra $X$ and $Y$, respectively. Since $X$ and $Y$ are each nonempty and compact, $X_{\mathrm{ex}}$ and $Y_{\mathrm{ex}}$ are each nonempty (Ref. 23).

We are now in a position to describe the four sets of conditions which give rise to the four special cases of problem $(\mathrm{P})$ of concern in this paper.

Case 1. Complete Efficiency. In this case, problem (MOLPY) is completely efficient. From an earlier observation, this case can be equivalently defined as the case where problem (MOLP) is completely efficient. Although the frequency of this case is unknown, it may be more common than was once thought, especially when $X$ has no interior (Ref. 24).

Case 2. Ideal Solution with Linear Dependence. In this case, the ideal solution $y^{I}$ for problem (MOLPY) belongs to $Y$, and $d$ is linearly dependent on the rows of $C$. It is rare for the condition $y^{I} \in Y$ to hold. On the other hand, $d$ may be linearly dependent on the rows of $C$ in certain common situations. For instance, $d$ is linearly dependent on the rows of $C$ when problem (P) represents the minimization of a criterion function $\left\langle c_{i}, x\right\rangle$ of problem (MOLP) over $X_{E}$.

Case 3. Bicriterion Case with Linear Dependence. In this case $p=2$ and $d$ is linearly dependent on the rows of $C$. When $p=2$, problem (MOLP) is called a bicriterion linear programming problem. Such problems are not uncommon and have received special attention in the literature (see Refs. 25-28, for instance). 
Case 4. Relaxation Case. In this case, an optimal solution $x^{*} \in X_{\mathrm{ex}}$ to the linear programming relaxation $\left(\mathrm{LP}_{\mathrm{UB}}\right)$ of problem $(\mathrm{P})$ exists which belongs to $X_{E}$.

For each case, we will describe a simple linear programming procedure which detects the case and solves problem $(\mathrm{P})$ under the special conditions of the case. Before doing so, however, we must present some theoretical results which serve as a basis for some of the procedures.

\section{Theoretical Prerequisites}

The following result gives a fundamental property of problem (P).

Theorem 3.1. Problem (P) has an optimal solution which belongs to $X_{\mathrm{ex}}$.

Proof. Since $X$ is nonempty and compact, $X_{E}$ is also nonempty and compact (Refs. 29-30). Therefore, problem (P) has at least one optimal solution. In addition, the compactness of $X$ implies that $X$ contains no lines (Ref. 23). From Theorem 4.5 in Ref. 10, it follows that problem (P) has at least one optimal solution which belongs to $X_{\mathrm{ex}}$.

We will use the following technical properties to help develop the remaining results of this section.

\section{Lemma 3.1.}

(a) For any $y^{\circ} \in Y_{E}$, if $x^{\circ} \in X$ satisfies $C x^{\circ}=y^{\circ}$, then $x^{\circ} \in X_{E}$.

(b) For any $x^{\circ} \in X_{E}$, if $y^{\circ}=C x^{\circ}$, then $y^{\circ} \in Y_{E}$.

Proof. Both (a) and (b) follow easily from the definitions of $X_{E}$ and $Y_{E}$.

Lemma 3.2. For any $y^{\circ} \in Y_{\text {ex }}$, there exists a point $x^{\circ} \in X_{\text {ex }}$ such that $C x^{\circ}=y^{\circ}$.

Proof. Suppose to the contrary that, if $C x=y^{\circ}$ for some $x \in X$, then $x \notin X_{\mathrm{ex}}$. Choose any $\bar{x} \in X$ such that $C \bar{x}=y^{\circ}$. Then, by assumption, $\bar{x} \notin X_{\mathrm{ex}}$. Since $X$ is compact, this implies that there exist points $z^{1}, z^{2}, \ldots, z^{q} \in X_{\mathrm{ex}}$ 
and scalars $\alpha_{1}, \alpha_{2}, \ldots, \alpha_{q}>0$ which sum to one such that

$$
\bar{x}=\sum_{i=1}^{q} \alpha_{i} z^{i}
$$

where $q \geqq 2$ (Ref. 23). Since $C \bar{x}=y^{\circ}$, this implies that

$$
y^{\circ}=\sum_{i=1}^{q} \alpha_{i} y^{i}
$$

where, for each $i=1,2, \ldots, q, y^{i}=C z^{i} \in Y$. For each $i=1,2, \ldots, q$, by assumption, since $z^{i} \in X_{\mathrm{ex}}, C z^{i} \neq y^{\circ}$ must hold. Therefore, $y^{i} \neq y^{\circ}$, $i=1,2, \ldots, q$.

Let

$$
Y \backslash\left\{y^{\circ}\right\}=\left\{y \in Y \mid y \neq y^{\circ}\right\} .
$$

Then it is easy to see that, since $y^{\circ}$ is an extreme point of $Y, Y \backslash\left\{y^{\circ}\right\}$ is a convex set. For each $i=1,2, \ldots, q$, since $y^{i} \in Y$ and $y^{i} \neq y^{\circ}$, it follows that $y^{i} \in Y \backslash\left\{y^{\circ}\right\}$. Therefore, any convex combination of the points $y^{i}$, $i=1,2, \ldots, q$, must lie in $Y \backslash\left\{y^{\circ}\right\}$. But since $Y$ is a convex set and the scalars $\alpha_{i}, i=1,2, \ldots, q$, are positive and sum to one, this contradicts (1), and the proof is complete.

The next result concerns the special conditions described in Case 2 in Section 2.

Theorem 3.2. Assume that the ideal solution $y^{I}$ for problem (MOLPY) belongs to $Y$ and that $d$ is linearly dependent on the rows of $C$. Then any $x^{\circ} \in X_{E}$ is an optimal solution for problem (P).

Proof. Since $d$ is linearly dependent on the rows of $C$, we may choose a vector $w \in R^{p}$ such that

$$
d^{T}=w^{T} C .
$$

Consider the problem (PY) given by

$$
\text { (PY) } \max \langle w, y\rangle, \quad \text { s.t. } y \in Y_{E} \text {. }
$$

By applying Theorem 3.1 to problem (PY), it follows that problem (PY) has an optimal solution which belongs to $Y_{\mathrm{ex}} \cap Y_{E}$. On the other hand, as mentioned earlier, since $y^{I} \in Y, Y_{E}=\left\{y^{n}\right\}$. Therefore, $y^{I} \in Y_{\mathrm{ex}}$ and $y^{I}$ is an optimal solution for problem (PY).

Choose any $x^{\circ} \in X_{E}$, and let $y^{\circ}=C x^{\circ}$. Then, by Lemma 3.1(b), $y^{\circ} \in Y_{E}$. Recalling that $Y_{E}=\left\{y^{I}\right\}$ whenever $y^{I} \in Y$, this implies that $y^{\circ}=y^{I}$. There- 
fore, $C x^{\circ}=y^{I}$. This implies, using (2), that

$$
\left\langle d, x^{\circ}\right\rangle=w^{T} C x^{\circ}=\left\langle w, y^{I}\right\rangle \text {. }
$$

Since $\left\langle w, y^{I}\right\rangle$ is a constant and $x^{\circ} \in X_{E}$ was arbitrarily chosen, (3) implies that any point $x^{\circ} \in X_{E}$ is an optimal solution for problem (P).

Remark 3.1. Notice in the proof of Theorem 3.2 that the point $x^{\circ} \in X_{E}$ satisfies $C x^{\circ}=y^{I}$. It follows that, under the conditions of Theorem 3.2 , any optimal solution $x^{\circ} \in X_{E}$ for problem (P) satisfies $C x^{\circ}=y^{I}$.

Remark 3.2. The assumption in Theorem 3.2 that $d$ is linearly dependent on the rows of $C$ cannot be omitted, as shown by the following example.

Example 3.1. Let

$$
X=\left\{x \in R^{3} \mid 0 \leqq x_{j} \leqq 1, j=1,2,3\right\},
$$

let $p=2$, and let $C$ and $d$ be given by

$$
C=\left[\begin{array}{lll}
1 & 0 & 0 \\
0 & 1 & 0
\end{array}\right], \quad d=\left[\begin{array}{l}
0 \\
0 \\
1
\end{array}\right],
$$

respectively. Then

$$
Y=\left\{\left(y_{1}, y_{2}\right) \in R^{2} \mid 0 \leqq y_{i} \leqq 1, i=1,2\right\},
$$

and $\left(y^{I}\right)^{T}=(1,1) \in Y$. The point $\left(x^{\circ}\right)^{T}=(1,1,0)$ satisfies $x^{\circ} \in X_{E}$. However, since

$$
X_{E}=\left\{x \in R^{3} \mid x_{1}=1, x_{2}=1,0 \leqq x_{3} \leqq I\right\},
$$

the unique optimal solution for problem $(\mathrm{P})$ is $\left(x^{*}\right)^{T}=(1,1,1)$. Therefore, the application of Theorem 3.2 to this example is invalid and yields the erroneous conclusion that $\left(x^{\circ}\right)^{T}=(1,1,0)$ is an optimal solution for problem (P). This is because $d$ is not linearly dependent on the rows of $C$ in this example.

The next result concerns the special conditions of Case 3 given in Section 2.

Theorem 3.3. Assume that $p=2$ and that $d$ is linearly dependent on the rows of $C$. Then, there exists an optimal solution $x^{*}$ for problem $(\mathrm{P})$ which belongs to $X_{\mathrm{ex}}$ and which is also an optimal solution to at least one 
of the linear programs $\left(\mathrm{LP}_{\mathrm{UB}}\right),\left(\mathrm{LP}_{1}\right)$, and $\left(\mathrm{LP}_{2}\right)$, where, for each $i=1,2$, problem $\left(L_{i}\right)$ is given by

$$
\left(\mathrm{L} \mathrm{P}_{\mathrm{i}}\right) \max \left\langle c_{i}, x\right\rangle, \quad \text { s.t. } x \in X .
$$

Proof. Let $w \in R^{2}$ satisfy $d^{T}=w^{T} C$. Since $p=2, Y$ is a nonempty, compact polyhedron in $R^{2}$. Consider the problem (PY) given by

$$
\text { (PY) } \max \langle w, y\rangle \text {, s.t. } y \in Y_{E} \text {. }
$$

Since $Y$ is compact and the feasible region of problem (PY) is $Y_{E}$, we may assume without loss of generality that, for some $k \geqq 1$,

$$
Y=\left\{y \in R^{2} \mid M y \leqq u, y \geqq 0\right\},
$$

where $M$ is a $k \times 2$ matrix and $u \in R^{k}$ (Ref. 31). By Theorem 3.1, we may choose an optimal solution $y^{*}$ for problem (PY) which belongs to $Y_{\mathrm{ex}}$. To prove the theorem, we will first show that $y^{*}$ maximizes at least one of $y_{1}$, $y_{2}$ and $\langle w, y\rangle$ over $Y$. There are four cases to consider.

Case 1. $y_{1}^{*}=y_{2}^{*}=0$. Then, since $Y \subseteq\left\{y \in R^{2} \mid y \geqq 0\right\}$ and $y^{*} \in Y_{E}$, $Y=\{0\}$. Therefore, in this case, $y^{*}$ maximizes both $y_{1}$ and $y_{2}$ over $Y$.

Case 2. $y_{1}^{*}>0$ and $y_{2}^{*}=0$. Suppose that $y \in Y$. Then, since $Y \subseteq\left\{y \in R^{2} \mid y \geqq 0\right\}, y_{2} \geqq y_{2}^{*}=0$. Since $y^{*} \in Y_{E}$, this implies that $y_{1} \leqq y_{1}^{*}$. Therefore, $y^{*}$ maximizes $y_{1}$ over $Y$.

Case 3. $y_{1}^{*}=0$ and $y_{2}^{*}>0$. Then, by a similar argument to the one given for Case 2, $y^{*}$ maximizes $y_{2}$ over $Y$.

Case 4. $y_{1}^{*}>0$ and $y_{2}^{*}>0$. Let $I_{k}$ denote the $k \times k$ identity matrix. From linear programming theory, since $y^{*}$ is an extreme point of $Y$, we may choose a basic feasible solution for the system

$$
\begin{aligned}
& M y^{1}+I_{k} y^{2}=u, \\
& y^{1}, y^{2} \geqq 0,
\end{aligned}
$$

which corresponds to $y^{1}=y^{*}$. After some possible column rearrangements, this basic feasible solution can be represented by the tableau

$$
T=\begin{array}{|cc|c|}
y^{N} & y^{B} \\
\hline-D & 0 & g \\
\hline A & I_{k} & b \\
\hline
\end{array}
$$

where $y^{N} \in R^{2}$ is the vector of nonbasic variables, $y^{B} \in R^{k}$ is the vector of basic variables in the basic feasible solution, and $y^{*}=g+D y^{N}=g$ (cf. 
Refs. 32-33, for instance). Let $D^{1}, D^{2} \in R^{2}$ denote the columns of $D$. Then either $b>0$ (nondegenerate case) or $b \geqq 0$ with $b_{i}=0$ for some $i \in\{1,2, \ldots, k\}$ (degenerate case).

Case 4a. Nondegenerate Case. Suppose that $y^{*}$ does not maximize either $y_{1}$ or $y_{2}$ over $Y$. We will use multiple objective linear programming theory to show that $y^{*}$ must maximize $\langle w, y\rangle$ over $Y$.

Since $b>0$, there exist $\alpha_{1}, \alpha_{2}>0$ such that, for each $q=1,2$, $z^{q}=g+\alpha_{q} D^{q}$ lies in the relative interior of $E^{q}$, where $E^{1}$ and $E^{2}$ are the two distinct edges of $Y$ which contain $y^{*}$. Since $y^{*}$ does not maximize $y_{1}$ or $y_{2}$ over $Y$, it follows from linear programming theory that (i) either $D_{1}^{1}>0$ or $D_{1}^{2}>0$ or both, and (ii) $D_{2}^{1}>0$ or $D_{2}^{2}>0$ or both. Since $y^{*} \in Y_{E}$, if $D_{1}^{1}>0$, then $D_{2}^{1}<0$. Similarly, if $D_{1}^{2}>0$, then $D_{2}^{2}<0$. Assume without loss of generality that $D_{1}^{1}>0$. Then, the preceding statements imply that $D_{2}^{1}<0, D_{1}^{2} \leqq 0$, and $D_{2}^{2}>0$.

From Ref. 32, since $y^{*} \in Y_{E}$, there exist $\bar{\lambda}_{1}, \bar{\lambda}_{2} \in R$ such that, with $\lambda_{1}=\bar{\lambda}_{1}$ and $\lambda_{2}=\bar{\lambda}_{2}$, the following inequalities are satisfied:

$$
\begin{aligned}
& \lambda_{1} D_{1}^{1}+\lambda_{2} D_{2}^{1} \leqq 0, \\
& \lambda_{1} D_{1}^{2}+\lambda_{2} D_{2}^{2} \leqq 0, \\
& \lambda_{1}, \lambda_{2} \geqq 1 .
\end{aligned}
$$

Since $D_{1}^{2} \leqq 0$ and $D_{2}^{2}>0$, this implies by (5) and (6) that $D_{1}^{2}<0$.

Consider edge $E^{1}$. From Ref. 6 , if (4) holds with equality with $\lambda_{1}=\bar{\lambda}_{1}$ and $\lambda_{2}=\bar{\lambda}_{2}$, then $E^{1} \subseteq Y_{E}$. If (4) does not hold with equality with $\lambda_{1}=\bar{\lambda}_{1}$ and $\lambda_{2}=\bar{\lambda}_{2}$, then since $D_{1}^{1}>0$ and $D_{1}^{2}<0$, there exists an $\epsilon>0$ such that, with $\lambda_{1}=\bar{\lambda}_{1}+\epsilon$ and $\lambda_{2}=\bar{\lambda}_{2},(4)-(6)$ are satisfied with (4) holding as an equality. Therefore, in this case, again $E^{1} \subseteq Y_{E}$ (Ref. 6). By a similar argument, with (5) replacing (4) and by increasing the value of $\lambda_{2}=\bar{\lambda}_{2}$, if necessary, it can be shown that $E^{2} \subseteq Y_{E}$.

Since $E^{1}, E^{2} \subseteq Y_{E}$, Theorem 4.6 in Ref. 10 implies that $y^{*}$ maximizes $\langle w, y\rangle$ over $Y$. Therefore, in this case, $y^{*}$ maximizes at least one of $y_{1}, y_{2}$ and $\langle w, y\rangle$ over $Y$.

Case 4b. Degenerate Case. Using an argument similar to that for Case $4 \mathrm{a}$, but with the technical adjustments required to account for degeneracy, it can be shown that, in this case, $y^{*}$ maximizes at least one of $y_{1}, y_{2}$ and $\langle w, y\rangle$ over $Y$. For brevity, the details of this argument are omitted.

It follows from Cases 1-4 that the optimal solution $y^{*} \in Y_{\mathrm{ex}}$ for problem (PY) maximizes at least one of $y_{1}, y_{2}$, and $\langle w, y\rangle$ over $Y$. By 
Lemma 3.2, we may choose a point $x^{*} \in X_{\text {ex }}$ such that $C x^{*}=y^{*}$. To complete the proof, we will show that $x^{*}$ is an optimal solution for problem $(\mathrm{P})$ and for at least one of problems $\left(\mathrm{LP}_{\mathrm{UB}}\right),\left(\mathrm{LP}_{1}\right)$, and $\left(\mathrm{LP}_{2}\right)$.

By Lemma 3.1(a), since $y^{*} \in Y_{E}, x^{*} \in X$, and $C x^{*}=y^{*}, x^{*} \in X_{E}$. Choose any optimal solution $x^{\circ}$ for problem (P), and let $C x^{\circ}=y^{\circ}$. Then

$$
\begin{aligned}
\left\langle d, x^{\circ}\right\rangle & =w^{T} C x^{\circ} \\
& =\left\langle w, y^{\circ}\right\rangle \\
& \leqq\left\langle w, y^{*}\right\rangle \\
& =w^{T} C x^{*} \\
& =\left\langle d, x^{*}\right\rangle,
\end{aligned}
$$

where the first and last equalities follow from $d^{T}=w^{T} C$, the second equality follows from $C x^{\circ}=y^{\circ}$, the inequality follows from $x^{\circ} \in X_{E}$, Lemma 3.1(b), and the optimality of $y^{*}$ in problem (PY), and the third equality follows from $C x^{*}=y^{*}$. Therefore, since $x^{*} \in X_{E}$ and $x^{\circ}$ is an optimal solution for problem (P), $x^{*}$ must also be an optimal solution for problem (P). Finally, since $y^{*}$ maximizes at least one of $y_{1}, y_{2}$, and $\langle w, y\rangle$ over $Y$ and $d^{T}=w^{T} C$, it easily follows that $x^{*}$ is an optimal solution to at least one of the problems $\left(L P_{U B}\right),\left(L_{1}\right)$, and $\left(L_{2}\right)$.

Remark 3.3. Assume that $p=2$ and $d$ is linearly dependent on the rows of $C$. Then, from Theorem 3.3, at least one extreme-point optimal solution for problem (P) can be found among the extreme-point optimal solutions to the linear programs $\left(\mathrm{LP}_{\mathrm{UB}}\right),\left(\mathrm{LP} \mathrm{P}_{1}\right)$, and $\left(\mathrm{LP}_{2}\right)$. However, as shown by the following example, not every extreme-point optimal solution for problem (P) need be an optimal solution to one of the problems $\left(\mathrm{LP}_{\mathrm{UB}}\right),\left(\mathrm{LP}_{1}\right)$, and $\left(\mathrm{LP}_{2}\right)$.

\section{Example 3.2. Let}

$$
X=\left\{x \in R^{3} \mid 0 \leqq x_{j} \leqq 1, j=1,2,3\right\},
$$

let $p=2$, and let $C$ and $d$ be given by

$$
C=\left[\begin{array}{rrr}
1 & 1 & 1 \\
0 & -1 & -1
\end{array}\right], \quad d=\left[\begin{array}{r}
-1 \\
0 \\
0
\end{array}\right],
$$

respectively. Then $d^{T}=w^{T} C$ holds for $w^{T}=(-1,-1)$, so that the assumptions of Theorem 3.3 hold. By observation,

$$
X_{E}=\left\{x \in R^{3} \mid x_{1}=1,0 \leqq x_{2}, x_{3} \leqq 1\right\},
$$


and any point in $X_{E}$ is an optimal solution for problem (P). Consider $\left(x^{\circ}\right)^{T}=(1,1,0)$ and $\left(x^{1}\right)^{T}=(1,0,1)$. Then $x^{\circ}, x^{1} \in X_{\text {ex }}$ and $x^{\circ}, x^{1}$ are optimal solutions for problem (P). However, for each $k=0,1, x^{k}$ is not an optimal solution to any of the linear programs $\left(\mathrm{LP}_{\mathrm{UB}}\right),\left(\mathrm{LP}_{1}\right)$, and $\left(\mathrm{LP}_{2}\right)$.

Remark 3.4. The proof of Theorem 3.3 illustrates the importance of using problem (MOLPY) to help analyze problem (P). In the proof, instead of attempting to directly show that there exists an optimal solution $x^{*} \in R^{n}$ for problem (P) which belongs to $X_{\mathrm{ex}}$ and optimally solves at least one of the three linear programs $\left(\mathrm{LP}_{\mathrm{UB}}\right),\left(\mathrm{LP}_{1}\right)$, and $\left(\mathrm{LP}_{2}\right)$, a simpler result involving problem (PY) is first shown. In particular, by applying certain results from multiple objective linear programming (cf. Refs. 6, 10, 32) to problems (MOLPY) and (PY), the proof shows that any optimal solution $y^{*} \in R^{2}$ for problem (PY) which belongs to $Y_{\mathrm{ex}}$ must also maximize one of $y_{1}, y_{2}$, and $\langle w, y\rangle$ over $Y$, where $w \in R^{2}$ satisfies $d^{T}=w^{T} C$. From this, the more complicated existence result stated in the theorem easily follows.

\section{Detecting and Solving the Four Special Cases}

In this section, based partly on the results developed in Section 3 , simple linear programming procedures are described for detecting and solving the four special cases of problem (P) given in Section 2 . We proceed on a case-by-case basis.

Case 1. Complete Efficiency. Both Benson (Ref. 24) and Benveniste (Ref. 34) have provided tests for detecting the complete efficiency of problem (MOLP). Benveniste's test, however, requires that $X$ have a nonempty interior, while Benson's does not. Therefore, to detect whether or not problem (MOLP) is completely efficient, we recommend using Benson's test.

Assume without loss of generality that

$$
X=\left\{x \in R^{n} \mid A x=b, x \geqq 0\right\},
$$

where $A$ is an $m \times n$ matrix and $b \in R^{m}$. Benson's test (Theorem 2 in Ref. 24) calls for finding the optimal value $\theta$ to the linear program ( $L P_{C E}$ ) given by

$$
\begin{array}{ll}
\left(\mathrm{LP}_{\mathrm{CE}}\right) \quad \min & \langle b, q\rangle, \\
\text { s.t. } & q^{T} A-z^{T} \geqq 0, \\
& -u^{T} C+r^{T} A-z^{T}=e^{T} C, \\
& u, z \geqq 0 .
\end{array}
$$

If $\theta=0$, problem (MOLP) is completely efficient. Otherwise, it is not. 
If complete efficiency holds, then any optimal solution to the linear program $\left(\mathrm{LP}_{\mathrm{UB}}\right)$ (cf. Section 2 ) is an optimal solution for problem $(\mathrm{P})$. We may therefore use the following linear programming procedures for this case.

Detection Procedure. Find the optimal value $\theta$ to the linear program $\left(\mathrm{LP}_{\mathrm{CE}}\right.$ ). If $\theta=0$, problem (MOLP) is completely efficient and Case 1 applies. If $\theta \neq 0$, problem (MOLP) is not completely efficient and Case 1 does not apply.

Solution Procedure. If $\theta=0$, find any optimal solution $x^{*}$ and the optimal value $\mathrm{UB}$ to the linear program $\left(\mathrm{LP}_{\mathrm{UB}}\right)$. Then $x^{*}$ is an optimal solution for problem (P) and $v=U B$.

Case 2. Ideal Solution with Linear Dependence. To detect whether or not $d$ is linearly dependent on the rows of $C$, it is necessary to decide whether or not a vector $w \in R^{p}$ exists such that $d^{T}=w^{T} C$. From duality theory of linear programming (Ref. 35), this may be accomplished by solving the linear program $\left(\mathrm{LP}_{\mathrm{D}}\right)$ given by

$$
\left(L P_{\mathrm{D}}\right) \max \langle d, u\rangle, \quad \text { s.t. } C u=0 .
$$

In particular, when the optimal value of problem $\left(\mathbf{L P}_{\mathrm{D}}\right)$ equals zero, $d$ is linearly dependent on the rows of $C$. Otherwise, problem $\left(\mathrm{LP}_{\mathrm{D}}\right)$ is feasible and unbounded, and $d$ is not linearly dependent on the rows of $C$.

To detect whether the ideal solution $y^{I}$ for problem (MOLPY) belongs to $Y$, one can solve, for instance, the linear program $\left(\mathrm{LP}_{\mathrm{I}}\right)$ given by

$$
\begin{aligned}
\left(\mathrm{LP}_{\mathrm{I}}\right) \max & \langle d, x\rangle, \\
\text { s.t. } & \left\langle c_{i}, x\right\rangle=y_{i}^{I}, \quad i=1,2, \ldots, p, \\
& x \in X .
\end{aligned}
$$

If problem $\left(\mathrm{LP}_{1}\right)$ is feasible, then any optimal solution $x^{\circ}$ satisfies $C x^{\circ}=y^{I} \in Y$ and $x^{\circ} \in X_{E}$. If problem $\left(\mathrm{LP}_{\mathrm{I}}\right)$ is infeasible, then the ideal solution $y^{I}$ for problem (MOLPY) does not belong to $Y$.

Based upon these observations and Theorem 3.2, we may use the following linear programming procedures for this case.

Detection Procedure.

(i) Solve the linear program $\left(L P_{D}\right)$. If problem $\left(L P_{D}\right)$ is feasible and unbounded, then Case 2 does not apply. Otherwise, problem $\left(\operatorname{LP}_{\mathrm{D}}\right)$ will have an optimal value of zero, and we continue to (ii) to see if Case 2 applies.

(ii) Determine whether or not the linear program $\left(\mathrm{LP}_{1}\right)$ is feasible. If problem $\left(\mathrm{LP}_{\mathrm{I}}\right)$ is feasible, then Case 2 applies. If it is not feasible, then Case 2 does not apply. 
Solution Procedure. When Case 2 is detected, find any optimal solution $x^{\circ}$ to the linear program $\left(\mathrm{LP}_{\mathrm{I}}\right)$. Then $x^{\circ}$ is an optimal solution for problem $(\mathrm{P})$ and $v=\left\langle d, x^{\circ}\right\rangle$.

Case 3. Bicriterion Case with Linear Dependence. Let $x^{\circ}$ be an arbitrary element of $X$. Then it is well known (see, for instance, Ref. 9) that $x^{\circ} \in X_{E}$ if and only if, with $x=x^{\circ}$, the linear program $\left(\mathrm{LP}_{\mathrm{x}}\right)$ given by

$$
\begin{array}{ll}
\left(\mathrm{LP}_{\mathrm{x}}\right) \quad \max & \langle e, s\rangle, \\
\text { s.t. } & C z-s=C x, \\
& z \in X, \\
& s \geqq 0,
\end{array}
$$

has an optimal value $v_{x}$ equal to zero, where $e \in R^{p}$ is the vector of ones. Based upon this result, part of the procedure for Case 2, and Theorem 3.3, we may use the following linear programming procedures for this case.

Detection Procedure. If $p \neq 2$, then Case 3 does not apply. If $p=2$, find the optimal value of the linear program $\left(L P_{D}\right)$. If this value equals zero, then Case 3 applies. Otherwise, it does not.

Solution Procedure. When Case 3 is detected, proceed as follows.

(i) Find every extreme point optimal solution to each of the linear programs $\left(L P_{U B}\right),\left(L P_{1}\right)$, and $\left(L P_{2}\right)$ [see Theorem 3.3 for the definitions of problems $\left.\left(\mathrm{LP}_{\mathrm{i}}\right), i=1,2\right]$. Let $\left\{x^{j} \mid j=1,2, \ldots, t\right\}$ represent the set of distinct extreme points found in this way.

(ii) For each $j=1,2, \ldots, t$, find the optimal value $v_{x j}$ to the linear program $\left(\mathrm{LP}_{\mathrm{x}}\right)$. Let $\left\{x^{j} \mid j \in J\right\}$ represent the subset of points of $\left\{x^{j} \mid j=1,2, \ldots, t\right\}$ for which $v_{x^{j}}=0$. Then any $x^{*} \in\left\{x^{j} \mid j \in J\right\}$ which satisfies

$$
\left\langle d, x^{*}\right\rangle=\max \left\{\langle d, x\rangle \mid x \in\left\{x^{j} \mid j \in J\right\}\right\}
$$

is an optimal solution for problem (P), and $v=\left\langle d, x^{*}\right\rangle$.

Remark 4.1. The solution procedure for Case 3 necessitates finding all extreme point optimal solutions to certain linear programming problems. This can be efficiently accomplished by using, for instance, the subroutines of ADBASE (Ref. 6).

Case 4. Relaxation Case. Based upon Theorem 3.1 and part of the procedure for Case 3 , we may use the following linear programming procedure for this case. 
Combined Detection-Solution Procedure.

(i) Find any extreme point optimal solution $x^{1}$ to the linear programming relaxation $\left(\mathrm{LP}_{\mathrm{UB}}\right)$ of problem (P). Set $j=1$.

(ii) Find the optimal value $v_{x j}$ to the linear program $\left(\mathrm{LP}_{\mathrm{x} i}\right)$. If $v_{x^{j}}=0$, then $x^{j}$ is an optimal solution for problem (P) and $v=U B=\left\langle d, x^{j}\right\rangle$. If $v_{x^{j}} \neq 0$, go to (iii).

(iii) Determine whether or not the linear program $\left(\mathrm{LP}_{\mathrm{UB}}\right)$ has an extreme point optimal solution distinct from each of $x^{1}, x^{2}, \ldots, x^{j}$. If not, then Case 4 does not apply. If so, find such a solution $x^{j+1}$, set $j=j+1$, and go to (ii).

Remark 4.2. The ADBASE program (Ref. 6) can be used to efficiently implement step (iii) of the procedure for Case 4 (cf. Remark 4.1).

\section{Conclusions}

The problem (P) of maximizing a linear function over the efficient set of a multiple objective linear program has important uses in multiple criteria decision making. Mathematically, problem (P) is generally a difficult global optimization problem which requires numerically intensive methods for its solution. In this paper, we have developed simple linear programming procedures for detecting and solving four special cases of problem $(\mathrm{P})$. These special-case procedures require very little computational effort in comparison to that required to solve the general problem (P). Therefore, when solving problem $(\mathrm{P})$, we recommend that they be used as screening devices to detect and solve the four special cases.

\section{References}

1. Evans, G. W., An Overview of Techniques for Solving Multiobjective Mathematical Programs, Management Science, Vol. 30, pp. 1268-1282, 1984.

2. Goicoechea, A., HAnsen, D. R., and Duckstein, L., Multiobjective Decision Analysis with Engineering and Business Applications, John Wiley and Sons, New York, New York, 1982.

3. Rosenthal, R. E., Principles of Multiobjective Optimization, Decision Sciences, Vol. 16, pp. 133-152, 1985.

4. Sawaragi, Y., Nakayama, H., and Tanino, T., Theory of Multiobjective Optimization, Academic Press, Orlando, Florida, 1985.

5. Stadler, W., A Survey of Multicriteria Optimization or the Vector Maximum Problem, Part I: 1776-1960, Journal of Optimization Theory and Applications, Vol. 29, pp. 1-52, 1979. 
6. Steuer, R. E., Multiple Criteria Optimization: Theory, Computation, and Application, John Wiley and Sons, New York, New York, 1986.

7. YU, P. L., Multiple Criteria Decision Making, Plenum, New York, New York, 1985.

8. YU, P. L., Multiple Criteria Decision Making: Five Basic Concepts, Optimization, Edited by G. L. Nemhauser, A. H. G. Rinnooy Kan, and M. J. Todd, North-Holland, Amsterdam, Holland, 1989.

9. ZelenY, M., Multiple Criteria Decision Making, McGraw-Hill, New York, New York, 1982.

10. Benson, H. P., Optimization over the Efficient Set, Journal of Mathematical Analysis and Applications, Vol. 98, pp. 562-580, 1984.

11. Benson, H. P., An Algorithm for Optimizing over the Weakly-Efficient Set, European Journal of Operational Research, Vol. 25, pp. 192-199, 1986.

12. PHILIP, J., Algorithms for the Vector Maximization Problem, Mathematical Programming, Vol. 2, pp. 207-229, 1972.

13. Dessouky, M. I., Ghiassi, M., and Davis, W. J., Estimates of the Minimum Nondominated Criterion Values in Multiple-Criteria Decision Making, Engineering Costs and Production Economics, Vol. 10, pp. 95-104, 1986.

14. Isermann, H., and Steuer, R. E., Computational Experience Concerning Payoff Tables and Minimum Criteria Values over the Efficient Set, European Journal of Operational Research, Vol. 33, pp. 91-97, 1987.

15. ReEves, G. R., and REID, R. C., Minimum Values over the Efficient Set in Multiple Objective Decision Making, European Journal of Operational Research, Vol. 36, pp. 334-338, 1988.

16. Weistroffer, H. R., Careful Use of Pessimistic Values Is Needed in Multiple Objectives Optimization, Operations Research Letters, Vol. 4, pp. 23-25, 1985.

17. HoRST, R., and TUY, H., Global Optimization: Deterministic Approaches, Springer-Verlag, Berlin, Germany, Second Edition, 1993.

18. Pardalos, P. M., and Rosen, J. B., Constrained Global Optimization: Algorithms and Applications, Springer-Verlag, Berlin, Germany, 1987.

19. Bolintineanu, S., Minimization of a Quasi-Concave Function over an Efficient Set, Mathematics Research Paper No. 90-15, La Trobe University, 1990.

20. Benson, H. P., An All-Linear Programming Relaxation Algorithm for Optimizing over the Efficient Set, Journal of Global Optimization, Vol. 1, pp. 83-104, 1991.

21. BENSON, H. P., A Finite, Nonadjacent Extreme Point Search Algorithm for Optimization over the Efficient Set, Journal of Optimization Theory and Applications, Vol. 73, pp. 47-64, 1992.

22. Dauer, J. P., Optimization over the Efficient Set Using an Active Constraint Approach, Zeitschrift für Operations Research, Vol. 35, pp. 185-195, 1991.

23. Rockafellar, R. T., Convex Analysis, Princeton University Press, Princeton, New Jersey, 1970.

24. BENSON, H. P., Complete Efficiency and the Initialization of Algorithms for Multiple Objective Programming, Operations Research Letters, Vol. 10, pp. 481-487, 1991. 
25. Geoffrion, A. M., Solving Bicriterion Mathematical Programs, Operations Research, Vol. 15, pp. 39-54, 1967.

26. BENSON, H. P., Vector Maximization with Two Objective Functions, Journal of Optimization Theory and Applications, Vol. 28, pp. 253-257, 1979.

27. WendelL, R. E., and LEE, D. N., Efficiency in Multiple Objective Optimization Problems, Mathematical Programming, Vol. 12, pp. 406-414, 1977.

28. ANEJA, Y. P., and NAIR, K. P. K., Bicriteria Transportation Problem, Management Science, Vol. 25, pp. 73-78, 1979.

29. Ecker, J. G., and KouAdA, I. A., Finding Efficient Points for Linear Multiple Objective Programs, Mathematical Programming, Vol. 18, pp. 375-377, 1975.

30. YU, P. L., and ZelENY, M., The Set of All Nondominated Solutions in Linear Cases and a Multicriteria Simplex Method, Journal of Mathematical Analysis and Applications, Vol. 49, pp. 430-468, 1975.

31. Shachtman, R., Generation of the Admissible Boundary of a Convex Polytope, Operations Research, Vol. 22, pp. 151-159, 1974.

32. ECKer, J. G., and KouAdA, I. A., Generating Maximal Efficient Faces for Multiple Objective Linear Programs, CORE Discussion Paper No. 7617, Université Catholique de Louvain, 1976.

33. Ecker, J. G., Hegner, N. S., and KouAdA, I. A., Generating All Maximal Efficient Faces for Multiple Objective Linear Programs, Journal of Optimization Theory and Applications, Vol. 30, pp. 353-381, 1980.

34. BENVENISTE, M., Testing for Complete Efficiency in a Vector Maximization Problem, Mathematical Programming, Vol. 12, pp. 285-288, 1977.

35. Murty, K. G., Linear Programming, John Wiley and Sons, New York, New York, 1983. 